\title{
Hepatic Dysfunction as Presenting Manifestation of Hodgkin's lymphoma
}

\section{Taylor DF*, Cho RS, Hall J, Walton D and Womeldorph CM}

\author{
Department of Gastroenterology and Department of Pathology, San Antonio Military Medical Center Fort Sam \\ Houston, TX
}

*Corresponding author: Taylor DF, Department of Gastroenterology and Department of Pathology, San Antonio Military Medical Center, Fort Sam Houston, TX 78234, United States, E-mail: douglas.f.taylor2. mil@mail.mil

Citation: Taylor DF, Cho RS, Hall J, Walton D, Womeldorph CM (2016) Hepatic Dysfunction as Presenting Manifestation of Hodgkin's lymphoma. J Gastroenterol Compl 1(1): 103

Received Date: January 04, 2016 Accepted Date: November 07, 2016 Published Date: November 10, 2016

\begin{abstract}
Hepatic dysfunction is rarely the presenting feature of malignancy, however it is important to recognize early on as the quick institution of chemotherapy could potentially reverse the liver disease. Without prompt therapy, hepatic failure is likely to occur which is characterized by encephalopathy, and coagulation deficits. There have been few case reports describing hepatic failure as the primary manifestation of Hodgkin's lymphoma, the malignancy itself precludes the possibility of liver transplant, and often typifies advanced disease. Our case describes gentlemen that presented with hepatic dysfunction secondary to Hodgkin's lymphoma, who was diagnosed before the onset of hepatic failure, and subsequently referred to oncology for chemotherapy.

Keywords: Hodgkin's lymphoma; Hepatic failure; Hepatic dysfunction
\end{abstract}

\section{Introduction}

Hepatic dysfunction is a potential complication of Hodgkin's disease that may be secondary to direct infiltration by the malignancy itself, or present as paraneoplastic manifestation of the malignancy. Hepatic dysfunction is concern as progression of the disease process may lead to hepatic failure. Fulminant hepatic failure is defined as an acute onset of liver disease compromising the liver's synthetic function. Typical findings include hepatic encephalopathy and prolongation of the INR $(>1.5)$ which occur within an 8 week time frame $[1,2]$. The most common precipitators of acute hepatic failure include viral hepatitis, medications, drugs, and toxins with less common causes including metabolic disorders and vascular disorders such as hepatic vein thrombosis [24]. Rarely is malignancy the presenting feature of liver dysfunction, but when the presentation does arise, the malignancy is typically advanced in stage, and not of hepatic origin [1-4]. This is significant as a history of malignancy precludes the patient from receiving a liver transplant [5], and if not addressed in a time, hepatic dysfunction may progress to hepatic failure. There have been numerous case reports in which a lymphoproliferative malignancy has involved the liver resulting in fulminant hepatic failure $[1,3,6-9]$. Hodgkin's disease (HD) is a lymph node based disease with 4 classic histologic subtypes with similar prognostic outcomes. Hepatic involvement in HD affects approximately 5-8\% of patients. Over half of patient's will present with right upper quadrant pain, jaundice, hepatomegaly, fevers, and night sweats with a generally poor prognosis.

\section{Materials and Methods}

Google scholar and PubMed were utilized with phrases such as "hepatic lymphoma," "Hodgkin's lymphoma and hepatic failure," "Hodgkin's lymphoma treatment," "hepatic dysfunction as presenting manifestation of hodgkin's lymphoma" which resulted in numerous articles, in these 8 case reports and 1 intergroup trial were selected. The articles were subsequently reviewed and data compared for write up of this case report.

\section{Case}

A 52 year old male with a history of hypertension and hyperlipidemia presented to the Brooke Army Medical Center emergency department complaining of stomach bloating, fevers, chills, night sweats, and unintentional weight loss of 40 lbs since January of this year. The patient was noted to have elevated liver associated enzymes at presentation primarily in a cholestatic pattern with an elevated alkaline phosphate, direct and total bilirubin, and INR. His physical examination was remarkable for hepatomegaly, no palpable spleen or palpable lymph nodes could be appreciated. Imaging was pursued with a right upper quadrant ultrasound 
showing mild homogenous echogenicity of the liver likely fatty infiltration versus fibrosis but could not exclude an underlying mass. Follow up imaging with a CT of the chest and abdomen revealed hepatosplenomegaly, a $2.5 \mathrm{~cm}$ RLL spiculated nodule concerning for metastatic process vs a primary lung malignancy, and diffuse retroperitoneal lymphadenopathy involving the peri-aortic, retro-crural, and left axilla. An MRI/MRCP with eovist was performed to rule out a liver mass and examine the extra hepatic biliary system. Additionally, a PET scan revealed left supraclavicular, left axillary, mediastinal, splenic hilum, and a diffuse retroperitoneal conglomerate all which were FDG avid concerning for an infiltrative process. The patient had biopsies taken of the liver and left axillary lymph node which were both consistent with classical Hodgkin's lymphoma. A bronchoalveolar lavage was performed but no evidence of malignant cells was present. The patient followed-up in the pulmonary clinic for an EBUS with biopsy for further evaluation of the spiculated lung mass. The results were rare large and atypical multinucleated cells classic for Hodgkin's lymphoma. The patient was subsequently referred to hematology oncology for further evaluation and management. His international prognostic score for Hodgkin's Lymphoma was 4 indicating a $61 \%$ chance of overall survival (Table 1).

\begin{tabular}{|c|c|}
\hline Lab test & Value \\
\hline Prothrombin time & 19.9 \\
\hline PTT & 64.2 \\
\hline INR & 1.7 \\
\hline Hemoglobin & 11.9 \\
\hline Hematocrit & 36 \\
\hline Platelets & 130 \\
\hline WBC & 9.3 \\
\hline Total protein & 6.9 \\
\hline Albumin & 3.1 \\
\hline AST & 52 \\
\hline ALT & 39 \\
\hline T-bili & 2.2 \\
\hline D-bili & 1.7 \\
\hline Alk-phos & 820 \\
\hline Lactate & 1.1 \\
\hline GGT & 225 \\
\hline CA 19-9 & 75.61 \\
\hline
\end{tabular}

Table 1: Initial Labs

The biopsy of the liver showed hepatic parenchyma with dense foci of portal lobular chronic inflammation with mostly small lymphocytes (Figure 1). There were large atypical cells scattered as well. Immunohistochemistry showed large atypical cells CD30 and CD15 positive. CD3 and CD5 were positive with small reactive T cells, CD20 positive for a few small B-cells. CD23 was positive for a few small lymphocytes. Many small reactive T-cells are positive for Ki67 and BCL2. In situ hybridization for EBV was negative. The findings were consistent with classical Hodgkin lymphoma.

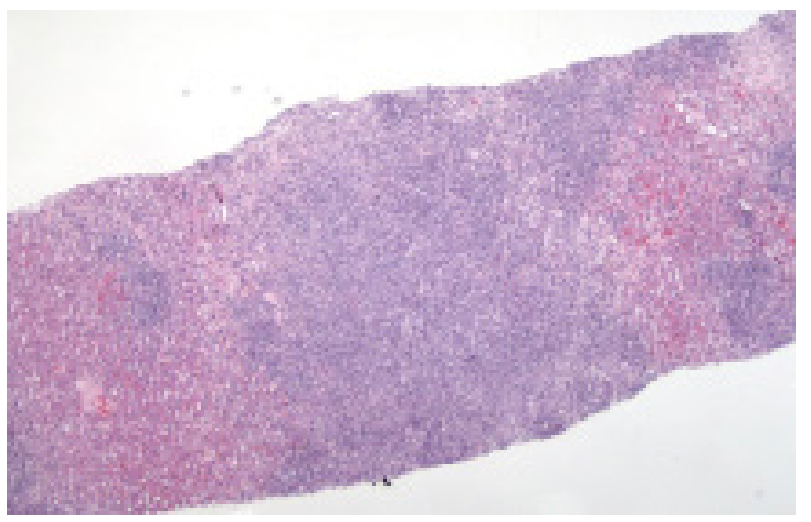

Figure 1: A low power view demonstrates a liver biopsy core with normal liver parenchyma interrupted by dense foci of chronic inflammation composed of mostly small lymphocytes

The axillary lymph node revealed rare large atypical cells and occasional large naked nuclei that could be seen with a hematopoietic or non-hematopoietic malignancy (Figure 2). 


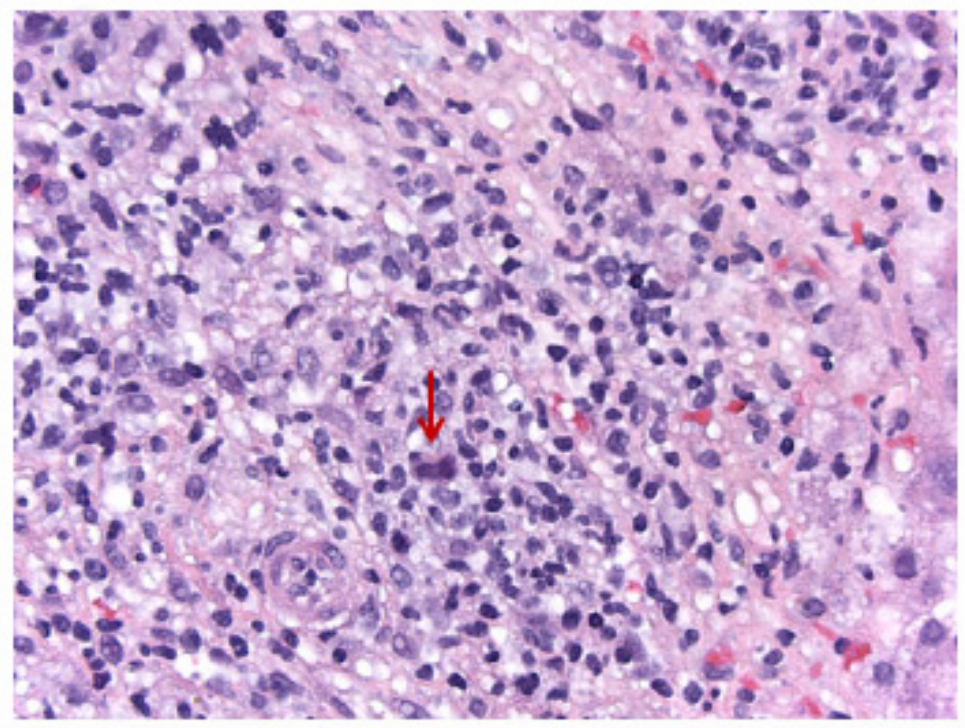

Figure 2: A higher power field demonstrates a large cell (red arrow) with eosinophilic cytoplasm and a dark, hyperchromatic nucleus. This cell is representative of other larger cells scattered amongst the smaller lymphocytes

Biopsy of lymph node station 4R showed a polymorphic lymphoid population with large mono-and multinucleated atypical cells with prominent macronucleoli suspicious for Reed-Sternberg (RS) cells. Broncho alveolar lavage identified no malignant cells.

\section{Discussion}

This case represents more hepatic dysfunction that overt liver failure given the effect on the patient's synthetic function but without overt hepatic encephalopathy. Hepatic dysfunction associated with malignancy has a broad range of effects on the liver ranging from mild dysfunction to fulminant hepatic failure. The effects on the liver could be secondary to direct infiltration by the lymphoma itself, or a paraneoplastic manifestation, in where the effects of the malignancy are due to humoral factors such as cytokines or the body's immune response resulting in damage to the liver. Hepatic dysfunction in Hodgkin's disease usually occurs late in the course of the disease, but is important to recognize as soon as possible as early intervention with chemotherapy could potentially alter the patient's clinical course $[1,2,4]$. However, Hodgkin's disease with hepatic involvement can prove difficult to treat if liver dysfunction is the primary presentation, and care is to be taken with drugs that are metabolized by the liver. Metastatic disease to the liver is not a common initial presentation for hepatic dysfunction or failure [3-6,8]. However, once the more common etiologies have been excluded it should be considered in the right clinical context. Imaging with ultrasound can be helpful with identifying infiltrating processes when mass-like lesions are present, but ultimately biopsy is necessary for histologic examination $[1,3,4]$. A lymph node biopsy showing mononuclear variants of RS cells in an appropriate background is sufficient to diagnose Hodgkin's disease. However liver biopsies require, then demonstration of classic multi-nucleated Reed-Sternberg cells for a diagnosis $[3,6]$.

Treatment of Hodgkin's disease affecting the liver differs based on the extent of hepatic involvement. Most cases are treated with chemotherapy, as the liver's tolerance to radiation is limited [3]. In our case, the patient followed up with the hematology/ oncology service and started receiving chemotherapy with ABVD (Adriamycin, bleomycin, vinblastine, and dacarbazine) which is first line treatment for Hodgkin's lymphoma, and has largely supplanted the previous MOPP (Mustargen, Oncovin, Procarbazine, Prednisone) regimen [9].

\section{Conclusion}

In short, in patient's presenting with altered hepatic function, hematologic malignancies should be considered in the differential diagnosis especially in the right clinical presentation and once other more common etiologies have been ruled out. Early recognition and tissue diagnosis is of utmost importance as early administration of chemotherapy could potentially reverse hepatic dysfunction.

\section{References}

1. Thompson DR, Thomas WF, Stone MJ, Polter DE (2001) Hepatic Failure as the Presenting Manifestation of malignant Lymphoma. Clinical Lymphoma 2: 123-8.

2. Karmacharya P, Bhandari N, Aryal MR, Pandit AA, Pathak R, et al. (2014) Before it crumbles: Fulminant Hepatic Failure secondary to Hodgkin's Lymphoma. J Community Hosp Intern Med Perspect 4: 25821.

3. Ortin X, Rodriguez-Luaces M, Bosch R, Lejeune M, Font L (2010) Acute liver failure as the first manifestation of very late relapsing of Hodgkin's disease. Hematology Rep 2: e5. 
4. Vardareli E, Dundar E, Aslan V, Fulbas Z (2004) Acute liver failure due to Hodgkin's Lymphoma. Med Princ Pract 13: 372-4.

5. Dourakis S, Tzemanakis E, Deutsch M, Kafiri G, Hadziyannis S (1999) Fulminant hepatic failure as a presenting paraneoplastic manifestation of Hodgkin's disease. Eur J Gastroenterol Hepatol 11: 1055-8.

6. Woolf KMW, Wei MC, Link MP, Arber DA, Warnke RA (2008) Nodular Lymphocyte-predominant Hodgkin Lymphoma presenting as Fulminant Hepatic Failure in a Pediatric Patient: A Case Report with Pathologic, Immunophenotypic and Molecular findings. Appl Immunohistochem Mol Morphol 16: 196-201.

7. Braud S, Portman B, Gimson AES, Williams, Roger (1982) Fulminant hepatic failure in non-hodgkin's lymphoma. Postgrad Med J 58: 301-4.

8. Rowbotham D, Wendon J, Williams R (1998) Acute liver failure secondary to hepatic infiltration: a single centre experience of 18 cases. Gut 42 : $576-80$.

9. Duggan D, Petroni G, Johnson JL, Glick JH, Fisher RI, et al. (2003) Randomized Comparison of ABVD and MOPP/ABV Hybride for the treatment of Advanced Hodgkin's Disease: Report of an intergroup trial. American society of clinical oncology 21: 607-14.



